Research Article

\title{
Study of Nonlinear Deformation of FCC-AuCuSi under Pressure by the Statistical Moment Method
}

\author{
Nguyen Quang Hoc $\mathbb{D}^{1},{ }^{1}$ Bui Duc Tinh $\mathbb{D},{ }^{1}$ Nguyen Duc Hien $\mathbb{D}^{2},{ }^{2}$ and Gelu Coman $\mathbb{D}^{3}$ \\ ${ }^{1}$ Hanoi National University of Education, 136 Xuan Thuy, Hanoi, Vietnam \\ ${ }^{2}$ Mac Dinh Chi High School, Chu Pah, Gia Lai, Vietnam \\ 3 "Dunarea de Jos" University of Galati, Galati, Romania \\ Correspondence should be addressed to Bui Duc Tinh; tinhbd@hnue.edu.vn
}

Received 19 October 2020; Revised 8 February 2021; Accepted 17 February 2021; Published 5 March 2021

Academic Editor: Hamed Akhavan

Copyright ( 92021 Nguyen Quang Hoc et al. This is an open access article distributed under the Creative Commons Attribution License, which permits unrestricted use, distribution, and reproduction in any medium, provided the original work is properly cited.

\begin{abstract}
In this work, we build the model and derive the theory of nonlinear deformation for substitutional alloy $\mathrm{AB}$ with interstitial atom $\mathrm{C}$ and face-centered cubic structure under pressure from the statistical moment method. The calculation results for FCC-AuCuSi are presented. We obtain the values of density of deformation energy, maximum real stress, limit of elastic deformation, and the stress-strain curve and compare the calculated results with experiments and other calculations.
\end{abstract}

\section{Introduction}

The study of elastic and thermodynamic properties of perfect ternary and binary interstitial alloys has also been attractive such as the theory of interstitial alloy [1], calculations from first principles, many-body potentials and dynamical dynamics for defects in metals, alloys and solid solutions [2-4] and elastic and thermodynamic properties of perfect ternary and binary interstitial alloys. We have studied the elastic deformation for body-entered cubic (BCC) and face-centered cubic (FCC) ternary and binary interstitial alloys under pressure by the statistical moment method (SMM) in [5-10].

The dependence of elastic and nonlinear deformations of materials on temperature, pressure, and concentration of components has very important role in predicting and understanding their interatomic interactions, strength, mechanical stability, phase transition mechanisms, and dynamical response. Silicides such as AuCuSi have attracted a lot of attention in recent years because of their functional applications and unusual physical properties. Gold silicide or gold silicon is one of the numerous metal alloys sold by American Elements under the trade name AE Alloys ${ }^{\mathrm{TM}}$.

The experimental data on the real stress and the limit of elastic deformation in the nonlinear deformation of pure metal $\mathrm{Au}$ are presented in [11-14].
Thermodynamic and mechanical properties of metals and interstitial alloys are studied by some theoretical methods and simulations. For example, Mehl and Papaconstantopoulos [15] applied a tight-binding (TB) scheme to extend first-principle calculations ( $a$ b initio) to regimes containing $10^{2}-10^{3}$ atoms in a unit cell and used two-center, nonorthogonal tight-binding parameters and on-site terms. Numerical calculations for many metals are compared with ab initio calculations and experiments. Deformation mechanisms in the mechanical response of nanoporous gold are investigated by molecular dynamics simulations [16]. In addition, in recent years, some researchers have considered factors affecting the structure, the phase transformation, and the crystallization process of alloys $\mathrm{AuCu}$ [17], $\mathrm{NiCu}[18,19]$, and $\mathrm{AgCu}[20]$.

In the present paper, we will study nonlinear deformation of FCC ternary alloy (substitutional alloy $A B$ with interstitial atoms $C$ ) under pressure by the statistical moment method (SMM) [21-24]. In Section 2, we build the model and theoretical calculations, and in Section 3, we carry out numerical calculations for alloy AuCuSi.

\section{Model and Theoretical Calculations}

In our model, for interstitial alloy $A C$ with FCC structure and concentration condition $c_{C}<<c_{A}\left(c_{A}=N_{A} / N\right.$ is the 
concentration of atoms $A, N_{A}$ is the number of atoms $A$, $c_{C}=N_{C} / N$ is the concentration of atoms $C, N_{C}$ is the number of atoms $C$, and $N=N_{A}+N_{C}$ is the total number of atoms of the alloy $A C$ ), the cohesive energy $u_{0}$ and the alloy parameters $k, \gamma_{1}, \gamma_{2}, \gamma$ ( $k$ is called the harmonic parameter and $\gamma_{1}, \gamma_{2}, \gamma$ are called anharmonic parameters) for the interstitial atom $\mathrm{C}$ in face centers of cubic unit cell in the approximation of two coordination spheres have the form [5-10]

$$
\begin{aligned}
u_{0 C}= & \frac{1}{2} \sum_{i=1}^{n_{i}} \varphi_{A C}\left(r_{i}\right)=3 \varphi_{A C}\left(r_{1 C}\right)+4 \varphi_{A C}\left(r_{2 C}\right), \\
r_{2 C}= & \sqrt{3} r_{1 C}, \\
k_{C}= & \frac{1}{2} \sum_{i}\left(\frac{\partial^{2} \varphi_{A C}}{\partial u_{i \beta}^{2}}\right)_{\mathrm{eq}}=\frac{\mathrm{d}^{2} \varphi_{A C}\left(r_{1 C}\right)}{\mathrm{d} r_{1 C}^{2}}+\frac{2}{r_{1 C}} \frac{\mathrm{d} \varphi_{A C}\left(r_{1 C}\right)}{\mathrm{d} r_{1 C}}+\frac{4}{3} \frac{\mathrm{d}^{2} \varphi_{A C}\left(r_{2 C}\right)}{\mathrm{d} r_{2 C}^{2}}+\frac{8}{3 r_{2 C}} \frac{\mathrm{d} \varphi_{A C}\left(r_{2 C}\right)}{\mathrm{d} r_{2 C}}, \\
\gamma_{C}= & 4\left(\gamma_{1 C}+\gamma_{2 C}\right), \\
\gamma_{1 C}= & \frac{1}{48} \sum_{i}\left(\frac{\partial^{4} \varphi_{A C}}{\partial u_{i \beta}^{4}}\right)_{\mathrm{eq}}=\frac{1}{24} \frac{\mathrm{d}^{4} \varphi_{A C}\left(r_{1 C}\right)}{\mathrm{d} r_{1 C}^{4}}+\frac{1}{4 r_{1 C}^{2}} \frac{\mathrm{d}^{2} \varphi_{A C}\left(r_{1 C}\right)}{\mathrm{d} r_{1 C}^{2}}-\frac{1}{4 r_{1 C}^{3}} \frac{\mathrm{d} \varphi_{A C}\left(r_{1 C}\right)}{\mathrm{d} r_{1 C}}+ \\
& +\frac{1}{54} \frac{\mathrm{d}^{4} \varphi_{A C}\left(r_{2 C}\right)}{\mathrm{d} r_{2 C}^{4}}+\frac{2}{9 r_{2 C}} \frac{\mathrm{d}^{3} \varphi_{A C}\left(r_{2 C}\right)}{\mathrm{d} r_{2 C}^{3}}-\frac{2}{9 r_{2 C}^{2}} \frac{\mathrm{d}^{2} \varphi_{A C}\left(r_{2 C}\right)}{\mathrm{d} r_{2 C}^{2}}+\frac{2}{9 r_{2 C}^{3}} \frac{\mathrm{d} \varphi_{A C}\left(r_{2 C}\right)}{\mathrm{d} r_{2 C}}, \\
& \left.+\frac{6}{9} \frac{\mathrm{d}^{4} \varphi_{A C}\left(r_{2 C}\right)}{\mathrm{d} r_{2 C}^{4}}+\frac{2}{3 r_{2 C}^{2}} \frac{\mathrm{d}^{2} \varphi_{A C}\left(r_{2 C}\right)}{\mathrm{d} r_{2 C}^{2}}-\frac{2}{3 r_{2 C}^{3}} \frac{\mathrm{d} \varphi_{A C}\left(r_{2 C}\right)}{\mathrm{d} r_{2 C}} \cdot \frac{\partial^{4} \varphi_{A C}}{\partial u_{i \alpha}^{2} \partial u_{i \beta}^{2}}\right)=\frac{\mathrm{d}^{3} \varphi_{A C}\left(r_{1 C}\right)}{2 r_{1 C}}-\frac{3}{4 r_{1 C}^{2}} \frac{\mathrm{d} \varphi_{A C}\left(r_{1 C}\right)}{\mathrm{d} r_{1 C}^{2}}+\frac{3}{4 r_{1 C}^{3}}+ \\
\gamma_{2 C} & \frac{1}{1 C} r_{1 C}
\end{aligned}
$$

The cohesive energy $u_{0}$ and the alloy parameters $k, \gamma_{1}, \gamma_{2}, \gamma$ for main metal atom $A_{1}$ in body center of cubic

unit cell in the approximation of three coordination spheres have the form [5-10]

$$
\begin{aligned}
u_{0 A_{1}} & =u_{0 A}+\varphi_{A C}\left(r_{1 A_{1}}\right), \\
k_{A_{1}} & =k_{A}+\frac{1}{2} \sum_{i}\left[\left(\frac{\partial^{2} \varphi_{A C}}{\partial u_{i \beta}^{2}}\right)_{\mathrm{eq}}\right]_{r=r_{1 A_{1}}}=k_{A}+\frac{\mathrm{d}^{2} \varphi_{A C}\left(r_{1 A_{1}}\right)}{\mathrm{d} r_{1 A_{1}}^{2}}, \\
\gamma_{A_{1}} & =4\left(\gamma_{1 A_{1}}+\gamma_{2 A_{1}}\right), \\
\gamma_{1 A_{1}} & =\gamma_{1 A}+\frac{1}{48} \sum_{i}\left[\left(\frac{\partial^{4} \varphi_{A C}}{\partial u_{i \beta}^{4}}\right)_{\mathrm{eq}}\right]_{r=r_{1 A_{1}}}=\gamma_{1 A}+\frac{1}{24} \frac{\mathrm{d}^{4} \varphi_{A C}\left(r_{1 A_{1}}\right)}{\mathrm{d} r_{1 A_{1}}^{4}}, \\
\gamma_{2 A_{1}} & =\gamma_{2 A}+\frac{6}{48} \sum_{i}\left[\left(\frac{\partial^{4} \varphi_{A C}}{\partial u_{i \alpha}^{2} \partial u_{i \beta}^{2}}\right)_{\mathrm{eq}}\right]_{r=r_{1 A_{1}}} \\
& =\gamma_{2 A}+\frac{1}{4 r_{1 A_{1}}} \frac{\mathrm{d}^{3} \varphi_{A C}\left(r_{1 A_{1}}\right)}{\mathrm{d} r_{1 A_{1}}^{3}}-\frac{1}{2 r_{1 A_{1}}^{2}} \frac{\mathrm{d}^{2} \varphi_{A C}\left(r_{1 A_{1}}\right)}{\mathrm{d} r_{1 A_{1}}^{2}}+\frac{1}{2 r_{1 A_{1}}^{3}} \frac{\mathrm{d} \varphi_{A C}\left(r_{1 A_{1}}\right)}{\mathrm{d} r_{1 A_{1}}} .
\end{aligned}
$$


The cohesive energy $u_{0}$ and the alloy parameters $k, \gamma_{1}, \gamma_{2}, \gamma$ for the main metal atom $A_{2}$ in corners of cubic unit cell in the approximation of three coordination spheres have the form [5-10]

$$
\begin{aligned}
u_{O A_{2}}= & u_{O A}+\varphi_{A C}\left(r_{1 A_{2}}\right), \\
k_{A_{2}}= & k_{A}+\frac{1}{2} \sum_{i}\left[\left(\frac{\partial^{2} \varphi_{A C}}{\partial u_{i \beta}^{2}}\right)_{\mathrm{eq}}\right]_{r=r_{1 A_{2}}}=k_{A}+\frac{1}{6} \frac{\mathrm{d}^{2} \varphi_{A C}\left(r_{1 A_{2}}\right)}{\mathrm{d} r_{1 A_{2}}^{2}}+\frac{23}{6 r_{1 A_{2}}} \frac{\mathrm{d} \varphi_{A C}\left(r_{1 A_{2}}\right)}{\mathrm{d} r_{1 A_{2}}}, \\
\gamma_{A_{2}}= & 4\left(\gamma_{1 A_{2}}+\gamma_{2 A_{2}}\right), \\
\gamma_{1 A_{2}}= & \gamma_{1 A}+\frac{1}{48} \sum_{i}\left[\left(\frac{\partial^{4} \varphi_{A C}}{\partial u_{i \beta}^{4}}\right)_{\mathrm{eq}}\right]_{r=r_{1 A_{2}}}=\gamma_{1 A}+\frac{1}{54} \frac{\mathrm{d}^{4} \varphi_{A C}\left(r_{1 A_{2}}\right)}{\mathrm{d} r_{1 A_{2}}^{4}}+\frac{2}{9 r_{1 A_{2}}} \frac{\mathrm{d}^{3} \varphi_{A C}\left(r_{1 A_{2}}\right)}{\mathrm{d} r_{1 A_{2}}^{3}} \\
& -\frac{2}{9 r_{1 A_{2}}^{2}} \frac{\mathrm{d}^{2} \varphi_{A C}\left(r_{1 A_{2}}\right)}{\mathrm{d} r_{1 A_{2}}^{2}}+\frac{2}{9 r_{1 A_{2}}^{3}} \frac{\mathrm{d} \varphi_{A C}\left(r_{1 A_{2}}\right)}{\mathrm{d} r_{1 A_{2}}}, \\
\gamma_{2 A_{2}}= & \gamma_{2 A}+\frac{6}{48} \sum_{i}\left[\left(\frac{\partial^{4} \varphi_{A C}}{\partial u_{i \alpha}^{2} \partial u_{i \beta}^{2}}\right)_{\mathrm{eq}}\right]_{r=r_{1 A_{2}}}=\gamma_{2 A}+\frac{1}{81} \frac{\mathrm{d}^{4} \varphi_{A C}\left(r_{1 A_{2}}\right)}{\mathrm{d} r_{1 A_{2}}^{4}} \\
& +\frac{4}{27 r_{1 A_{2}}} \frac{\mathrm{d}^{3} \varphi_{A C}\left(r_{1 A_{2}}\right)}{\mathrm{d} r_{1 A_{2}}^{3}}+\frac{14}{27 r_{1 A_{2}}^{2}} \frac{\mathrm{d}^{2} \varphi_{A C}\left(r_{1 A_{2}}\right)}{\mathrm{d} r_{1 A_{2}}^{2}}-\frac{14}{27 r_{1 A_{2}}^{3}} \frac{\mathrm{d} \varphi_{A C}\left(r_{1 A_{2}}\right)}{\mathrm{d} r_{1 A_{2}}},
\end{aligned}
$$

where $\phi_{A C}$ is the interaction potential between atoms $A$ and $C, r_{1 X}=r_{01 X}+y_{0 X}(T)$ is the nearest neighbor distance between the atom $X\left(X=A, A_{1}, A_{2}, C\right)(A$ in clean metal and $A_{1}, A_{2}$, and $C$ in interstitial alloy $A C$ ) and other atoms at temperature $T, r_{01 X}$ is the nearest neighbor distance between the atom $X$ and other atoms at $T=0 \mathrm{~K}$ and is determined from the minimum condition of the cohesive energy $u_{0 X}$, and $y_{0 X}(T)$ is the displacement of atom $X$ from equilibrium position at temperature T. $u_{0 A}, k_{A}, \gamma_{1 A}, \gamma_{2 A}$ are the corresponding quantities in the clean metal $A$ with FCC structure in the approximation of two coordination spheres and have the form $[21,22]$

$$
\begin{aligned}
u_{0 A}= & 6 \varphi_{A A}\left(r_{1 A}\right)+3 \varphi_{A A}\left(r_{2 A}\right), \\
r_{2 A}= & \sqrt{2} r_{1 A}, \\
k_{A}= & 2 \frac{\mathrm{d}^{2} \varphi_{A A}\left(r_{1 A}\right)}{\mathrm{d} r_{1 A}^{2}}+\frac{4}{r_{1 A}} \frac{\mathrm{d} \varphi_{A A}\left(r_{1 A}\right)}{\mathrm{d} r_{1 A}}+\frac{\mathrm{d}^{2} \varphi_{A A}\left(r_{2 A}\right)}{\mathrm{d} r_{2 A}^{2}}+\frac{2}{r_{2 A}} \frac{\mathrm{d} \varphi_{A A}\left(r_{2 A}\right)}{\mathrm{d} r_{2 A}}, \\
\gamma_{1 A}= & \frac{1}{24} \frac{\mathrm{d}^{4} \varphi_{A A}\left(r_{1 A}\right)}{\mathrm{d} r_{1 A}^{4}}+\frac{1}{4 r_{1 A}} \frac{\mathrm{d}^{3} \varphi_{A A}\left(r_{1 A}\right)}{\mathrm{d} r_{1 A}^{3}}-\frac{1}{8 r_{1 A}^{2}} \frac{\mathrm{d}^{2} \varphi_{A A}\left(r_{1 A}\right)}{\mathrm{d} r_{1 A}^{2}}+\frac{1}{8 r_{1 A}^{3}} \frac{\mathrm{d} \varphi_{A A}\left(r_{1 A}\right)}{\mathrm{d} r_{1 A}} \\
& +\frac{1}{24} \frac{\mathrm{d}^{4} \varphi_{A A}\left(r_{2 A}\right)}{\mathrm{d} r_{2 A}^{4}}+\frac{1}{4 r_{2 A}^{2}} \frac{\mathrm{d}^{2} \varphi_{A A}\left(r_{2 A}\right)}{\mathrm{d} r_{2 A}^{2}}-\frac{1}{4 r_{2 A}^{3}} \frac{\mathrm{d} \varphi_{A A}\left(r_{2 A}\right)}{\mathrm{d} r_{2 A}}, \\
\gamma_{2 A}= & \frac{1}{48} \frac{\mathrm{d}^{4} \varphi_{A A}\left(r_{1 A}\right)}{\mathrm{d} r_{1 A}^{4}}+\frac{7}{8 r_{1 A}} \frac{\mathrm{d}^{3} \varphi_{A A}\left(r_{1 A}\right)}{\mathrm{d} r_{1 A}^{3}}-\frac{31}{16 r_{1 A}^{2}} \frac{\mathrm{d}^{2} \varphi_{A A}\left(r_{1 A}\right)}{\mathrm{d} r_{1 A}^{2}}+\frac{31}{16 r_{1 A}^{3}} \frac{\mathrm{d} \varphi_{A A}\left(r_{1 A}\right)}{\mathrm{d} r_{1 A}} \\
& +\frac{1}{2 r_{2 A}} \frac{\mathrm{d}^{3} \varphi_{A A}\left(r_{2 A}\right)}{\mathrm{d} r_{2 A}^{3}}-\frac{9}{8 r_{2 A}^{2}} \frac{\mathrm{d}^{2} \varphi_{A A}\left(r_{2 A}\right)}{\mathrm{d} r_{2 A}^{2}}+\frac{9}{8 r_{2 A}^{3}} \frac{\mathrm{d} \varphi_{A A}\left(r_{2 A}\right)}{\mathrm{d} r_{2 A}} .
\end{aligned}
$$


The equations of state for FCC interstitial alloy at temperature $T$ and pressure $P$ and at $0 \mathrm{~K}$ and pressure $P$ are written in the form $[5,6,8-10,23]$

$$
\begin{aligned}
P v & =-r_{1}\left[\frac{1}{6} \frac{\partial u_{o}}{\partial r_{1}}+\theta x c t h x \frac{1}{2 k} \frac{\partial k}{\partial r_{1}}\right], \\
v & =\frac{\sqrt{2} r_{1}^{3}}{2} \\
P v & =-r_{1}\left(\frac{1}{6} \frac{\partial u_{0}}{\partial r_{1}}+\frac{\hbar \omega_{0}}{4 k} \frac{\partial k}{\partial r_{1}}\right) .
\end{aligned}
$$

From that, we can calculate the nearest neighbor distance $r_{1 X}(P, 0)\left(X=A, A_{1}, A_{2}, C\right)$, the parameters $k_{X}(P, 0), \gamma_{1 X}(P, 0), \gamma_{2 X}(P, 0), \gamma_{X}(P, 0)$, the displacement $y_{0 X}(P, T)$ of atom $X$ from equilibrium position as in [21], the nearest neighbor distance $r_{1 X}(P, T)$, and the mean nearest neighbor distance between two atoms $A$ in alloy $\overline{r_{1 A}(P, T)}$ as follows $[5,6,8-10]$ :

$$
\begin{aligned}
r_{1 C}(P, T) & =r_{1 C}(P, 0)+y_{A_{1}}(P, T), \\
r_{1 A}(P, T) & =r_{1 A}(P, 0)+y_{A}(P, T), \\
r_{1 A_{1}}(P, T) & \approx r_{1 B}(P, T), \\
r_{1 A_{2}}(P, T) & =r_{1 A_{2}}(P, 0)+y_{C}(P, T), \\
\overline{r_{1 A}(P, T)} & =\overline{r_{1 A}(P, 0)}+\overline{y(P, T)}, \\
\overline{r_{1 A}(P, 0)} & =\left(1-c_{C}\right) r_{1 A}(P, 0)+c_{C} r_{1 A}^{\prime}(P, 0), r_{1 A}^{\prime}(P, 0)=\sqrt{3} r_{1 C}(P, 0), \\
\overline{y(P, T)} & =\left(1-15 c_{C}\right) y_{A}(P, T)+c_{C} y_{C}(P, T)+6 c_{C} y_{A_{1}}(P, T)+8 c_{C} y_{A_{2}}(P, T) .
\end{aligned}
$$

The Helmholtz free energy of FCC interstitial alloy $A C$ with the condition $c_{C}<<c_{A}$ is determined by [5-10,21]

$$
\begin{aligned}
\psi_{A C}= & \left(1-15 c_{C}\right) \psi_{A}+c_{C} \psi_{C}+6 c_{C} \psi_{A_{1}}+8 c_{C} \psi_{A_{2}}-T S_{c}, \\
\psi_{X} \approx & U_{0 X}+\psi_{0 X}+3 N\left\{\frac{\theta^{2}}{k_{X}^{2}}\left[\gamma_{2 X} Y_{X}^{2}-\frac{2 \gamma_{1 X}}{3}\left(1+\frac{Y_{X}}{2}\right)\right]\right. \\
& +\frac{2 \theta^{3}}{k_{X}^{4}}\left\{\left[\frac{4}{3} \gamma_{2 X} Y_{X}\left(1+\frac{Y_{X}}{2}\right)-2\left[\gamma_{1 X}^{2}+2 \gamma_{1 X} \gamma_{2 X}\right]\right.\right. \\
& \left.\left(1+\frac{Y_{X}}{2}\right)\left(1+Y_{X}\right)\right], \\
\psi_{0 X}= & 3 N \theta\left[x_{X}+\ln \left(1-e^{-2 x_{X}}\right)\right], \\
Y_{X} \equiv & x_{X} \operatorname{coth} x_{X},
\end{aligned}
$$

where $\psi_{X}$ is the Helmholtz free energy of one atom $X, U_{0 X}$ is the cohesive energy, and $S_{c}$ is the configurational entropy of FCC interstitial alloy $A C$.

The nearest neighbor distances between two atoms in alloy $A C$ after deformation have the form [9]

$$
\begin{aligned}
r_{01 X}^{F} & =r_{01 X}(1+\varepsilon), \\
r_{1 X}^{F}(P, 0) & =r_{1 X}(P, 0)\left(1+2 \varepsilon+\varepsilon^{2}\right) \\
r_{1 C}^{F}(P, T) & =r_{01 C}^{F}(P, 0)+y_{A_{1}}^{F}(P, 0), \\
r_{1 A}^{F}(P, 0) & =r_{01 A}^{F}(P, 0)+y_{A}^{F}(P, T), \\
r_{1 A_{1}}^{F}(P, T) & \approx r_{1 C}^{F}(P, T), \\
r_{1 A_{2}}^{F}(P, T) & =r_{01 A_{2}}^{F}(P, 0)+y_{C}^{F}(P, T) .
\end{aligned}
$$

The mean nearest neighbor distances between two atoms $A$ in interstitial alloy $A C$ at pressure $P$ and temperature $T$ after deformation have the form [9]

$$
\begin{aligned}
& \overline{r_{1 A}^{A C F}(P, T)}=\overline{r_{01 A}^{A C F}(P, 0)}+\overline{y^{A C F}(P, T)}, \\
& \overline{r_{01 A}^{A C F}(P, 0)}=\left[\left(1-c_{C}\right) r_{01 A}^{F}(P, 0)+c_{C} r_{01 A}^{\prime A C F}(P, 0)\right]\left(1+2 \varepsilon+\varepsilon^{2}\right) \text {, } \\
& r_{01 A}^{\prime F}(P, 0)=\sqrt{3} r_{01 C}^{F}(P, 0) \text {, } \\
& \overline{y^{A C F}(P, T)}=\left(1-15 c_{C}\right) y_{A}^{F}(P, T)+c_{C} y_{C}^{F}(P, T)+6 c_{C} y_{A_{1}}^{F}(P, T)+8 c_{C} y_{A_{2}}^{F}(P, T) \text {. }
\end{aligned}
$$


The mean nearest neighbor distances between two atoms $A$ in substitutional alloy $A B$ with interstitial atoms $C$ at pressure $P$ and temperature $T$ and at pressure $P$ and temperature $T=0 \mathrm{~K}$ after deformation have the form $[5,6,9]$

$$
\begin{aligned}
& a_{A B C}^{F}=c_{A C} a_{A C}^{F} \frac{B_{T A C}^{F}}{B_{T}^{\text {bccF }}}+c_{B} a_{B}^{F} \frac{B_{T B}^{F}}{\overline{B_{T}^{F}}}, \\
& \overline{B_{T}^{F}}=c_{A C} B_{T A C}^{F}+c_{B} B_{T B}^{F}, \\
& c_{A C}=c_{A}+c_{C}, \\
& a_{A B C}^{F} \equiv \overline{r_{1 A}^{A B C F}(P, T)}, \\
& a_{A C}^{F} \equiv \overline{r_{1 A}^{A C F}(P, T)}, \\
& a_{B}^{F} \equiv r_{1 B}^{F}(P, T), \\
& B_{T A C}^{F}=\frac{1}{\chi_{T A C}^{F}} \\
& B_{T B}^{F}=\frac{1}{\chi_{T B}^{F}} \\
& \left(\frac{\partial^{2} \psi_{A C}^{F}}{\partial a_{A C}^{F 2}}\right)_{T} \approx\left(1-15 c_{C}\right)\left(\frac{\partial^{2} \psi_{A}^{F}}{\partial a_{A}^{F 2}}\right)_{T}+c_{C}\left(\frac{\partial^{2} \psi_{C}^{F}}{\partial a_{C}^{F 2}}\right)_{T}+6 c_{C}\left(\frac{\partial^{2} \psi_{A_{1}}^{F}}{\partial a_{A_{1}}^{F 2}}\right)_{T}+8 c_{C}\left(\frac{\partial^{2} \psi_{A_{2}}^{F}}{\partial a_{A_{2}}^{F 2}}\right)_{T}, \\
& \frac{1}{3 N}\left(\frac{\partial^{2} \psi_{X}^{F}}{\partial a_{X}^{F 2}}\right)_{T}=\frac{1}{6} \frac{\partial^{2} u_{0 X}^{F}}{\partial a_{X}^{F 2}}+\frac{\hbar \omega_{X}^{F}}{4 k_{X}^{F}}\left[\frac{\partial^{2} k_{X}^{F}}{\partial a_{X}^{F 2}}-\frac{1}{2 k_{X}^{F}}\left(\frac{\partial k_{X}^{F}}{\partial a_{X}^{F}}\right)^{2}\right], \\
& X=A, A_{1}, A_{2}, B, C \text {, } \\
& a_{0 A B C}^{F}=c_{A C} a_{0 A C}^{F} \frac{B_{0 T A C}^{F}}{\overline{B_{0 T}^{F}}}+c_{B} a_{0 B}^{F} \frac{B_{0 T B}^{F}}{\overline{B_{0 T}^{F}}}, \\
& \overline{B_{0 T}^{F}}=c_{A C} B_{0 T A C}^{F}+c_{B} B_{0 T B}^{F} \text {, } \\
& a_{0 A B C}^{F} \equiv \overline{r_{01 A}^{A B C F}(P, 0)}, \\
& a_{0 A C}^{F} \equiv \overline{r_{01 A}^{A C F}(P, 0)}, \\
& a_{0 B}^{F} \equiv r_{01 B}^{F}(P, 0) \text {. }
\end{aligned}
$$

The Helmholtz free energy of alloy $\mathrm{ABC}$ before and after deformation with the condition $c_{C}<<c_{B}<<c_{A}$ is determined by $[5,6,9]$

$$
\begin{aligned}
\psi_{A B C} & =\psi_{A C}+c_{B}\left(\psi_{B}-\psi_{A}\right)+T S_{c}^{A C}-T S_{c}^{A B C}, \\
\psi_{A C} & =\left(1-15 c_{C}\right) \psi_{A}+c_{C} \psi_{C}+6 c_{C} \psi_{A_{1}}+8 c_{C} \psi_{A_{2}}-T S_{c}^{A C}, \\
\psi_{A B C}^{F} & =\psi_{A C}^{F}+c_{B}\left(\psi_{B}^{F}-\psi_{A}^{F}\right)+T S_{c}^{A C F}-T S_{c}^{A B C F}, \\
\psi_{A C}^{F} & =\left(1-15 c_{C}\right) \psi_{A}^{F}+c_{C} \psi_{C}^{F}+6 c_{C} \psi_{A_{1}}^{F}+8 c_{C} \psi_{A_{2}}^{F}-T S_{c}^{A C F} .
\end{aligned}
$$

The relationship between the stress and the deformation in nonlinear deformation is given by [9]

$$
\sigma_{1 A B C}=\sigma_{0 A B C} \frac{\varepsilon^{\alpha_{A B C}}}{1+\varepsilon}
$$

where $\sigma_{0 A B C}$ and $\alpha_{A B C}$ are constants for every alloy.

The density of deformation energy can be written in the form [9] 


$$
\begin{aligned}
f_{A B C}(\varepsilon)= & \frac{1}{N}\left(1-c_{A}\right)\left\{\psi_{A}\left(\frac{1}{v_{A B C}^{F}}-\frac{1}{v_{A B C}}\right)+\frac{2 \varepsilon r_{01 A}^{F}}{v_{A B C}^{F}}\left(\frac{\partial \psi_{A}^{F}}{\partial r_{1 A}^{F}}\right)_{T}+\frac{\varepsilon^{2}}{2 v_{A B C}^{F}}\left[\left(\frac{\partial^{2} \psi_{A}^{F}}{\partial r_{1 A}^{F 2}}\right)_{T}\left(2 r_{01 A}^{F}\right)^{2}+\left(\frac{\partial \psi_{A}^{F}}{\partial r_{1 A}^{F}}\right)_{T} 2 r_{01 A}^{F}\right]\right\}+ \\
& +\frac{c_{C}}{N}\left\{\psi_{B}\left(\frac{1}{v_{A B C}^{F}}-\frac{1}{v_{A B C}}\right)+\frac{2 \varepsilon r_{01 B}^{F}}{v_{A B C}^{F}}\left(\frac{\partial \psi_{B}^{F}}{\partial r_{1 B}^{F}}\right)_{T}+\frac{\varepsilon^{2}}{2 v_{A B C}^{F}}\left[\left(\frac{\partial^{2} \psi_{B}^{F}}{\partial r_{1 B}^{F 2}}\right)_{T}\left(2 r_{01 B}^{F}\right)^{2}+\left(\frac{\partial \psi_{B}^{F}}{\partial r_{1 B}^{F}}\right)_{T} 2 r_{01 B}^{F}\right]\right\}+ \\
& +\frac{c_{A_{1}}}{N}\left\{\psi_{A_{1}}\left(\frac{1}{v_{A B C}^{F}}-\frac{1}{v_{A B C}}\right)+\frac{2 \varepsilon r_{01 A_{1}}^{F}}{v_{A B C}^{F}}\left(\frac{\partial \psi_{A_{1}}^{F}}{\partial r_{1 A_{1}}^{F}}\right)_{T}+\frac{\varepsilon^{2}}{2 v_{A B C}^{F}}\left[\left(\frac{\partial^{2} \psi_{A_{1}}^{F}}{\partial r_{1 A_{1}}^{F 2}}\right)_{T}\left(2 r_{01 A_{1}}^{F}\right)^{2}+\left(\frac{\partial \psi_{A_{1}}^{F}}{\partial r_{1 A_{1}}^{F}}\right)_{T} 2 r_{01 A_{1}}^{F}\right]\right\}+ \\
& +\frac{c_{A_{2}}}{N}\left\{\psi_{A_{2}}\left(\frac{1}{v_{A B C}^{F}}-\frac{1}{v_{A B C}}\right)+\frac{2 \varepsilon r_{01 A_{2}}^{F}}{v_{A B C}^{F}}\left(\frac{\partial \psi_{A_{2}}^{F}}{\partial r_{1 A_{2}}^{F}}\right)_{T}+\frac{\varepsilon^{2}}{2 v_{A B C}^{F}}\left[\left(\frac{\partial^{2} \psi_{A_{2}}^{F}}{\partial r_{1 A_{2}}^{F 2}}\right)_{T}\left(2 r_{01 A_{2}}^{F}\right)^{2}+\left(\frac{\partial \psi_{A_{2}}^{F}}{\partial r_{1 A_{2}}^{F}}\right) 2 r_{T}^{F}\right]\right\}+ \\
& +\frac{c_{B}}{N}\left\{\psi_{B}\left(\frac{1}{v_{A B C}^{F}}-\frac{1}{v_{A B C}}\right)+\frac{2 \varepsilon r_{01 B}^{F}}{v_{A B C}^{F}}\left(\frac{\partial \psi_{B}^{F}}{\partial r_{1 B}^{F}}\right)_{T}+\frac{\varepsilon^{2}}{2 v_{A B C}^{F}}\left[\left(\frac{\partial^{2} \psi_{B}^{F}}{\partial r_{1 B}^{F 2}}\right)_{T}\left(2 r_{01 B}^{F}\right)^{2}+\left(\frac{\partial \psi_{B}^{F}}{\partial r_{1 B}^{F}}\right)_{T} 2 r_{01 B}^{F}\right]\right\}- \\
& -\frac{c_{B}}{N}\left\{\psi_{A}\left(\frac{1}{v_{A B C}^{F}}-\frac{1}{v_{A B C}}\right)+\frac{2 \varepsilon r_{01 A}^{F}}{v_{A B C}^{F}}\left(\frac{\partial \psi_{A}^{F}}{\partial r_{1 A}^{F}}\right)_{T}+\frac{\varepsilon^{2}}{2 v_{A B C}^{F}}\left[\left(\frac{\partial^{2} \psi_{A}^{F}}{\partial r_{1 A}^{F 2}}\right)_{T}\left(2 r_{01 A}^{F}\right)^{2}+\left(\frac{\partial \psi_{A}^{F}}{\partial r_{1 A}^{F}}\right)_{T} 2 r_{01 A}^{F}\right]\right\} .
\end{aligned}
$$

When the deformation rate is constant, the density of deformation energy of alloy is determined by [9]

$$
f_{A B C}(\varepsilon)=C_{A B C} \cdot \sigma_{A B C} \cdot \varepsilon,
$$

where $C_{A B C}$ is the proportional factor. At the maximum value of the density of deformation energy, we have

$$
f_{A B C}\left(\varepsilon_{A B C}^{F}\right)=f_{A B C \max }=C_{A B C} \sigma_{A B C \max } \varepsilon_{A B C}^{F} .
$$

The maximum value of stress $\sigma_{A B C \max }$ and the maximum real stress $\sigma_{1 A B C \max }$ are [9]

$$
\begin{aligned}
\sigma_{A B C \max } & =\frac{f_{A B C \max }}{C_{A B C} \varepsilon_{A B C}^{F}}, \\
\sigma_{1 A B C \max }= & \frac{\sigma_{A B C \max }}{1+\varepsilon_{A B C}^{F}}=\frac{f_{A B C \max }}{C_{A B C} \varepsilon_{A B C}^{F}\left(1+\varepsilon_{A B C}^{F}\right)} .
\end{aligned}
$$

From the maximum condition of stress $\left(\partial \sigma_{1 A B C} / \partial \varepsilon\right)_{\varepsilon_{A B C}^{F}}=0$, we derive the deformation $\varepsilon_{A B C}^{F}$ corresponding to the maximum value of real stress as follows [9]:

$$
\varepsilon_{A B C}^{F}=\frac{\alpha_{A B C}}{1-\alpha_{A B C}} \Rightarrow \sigma_{1 A B C \max }=\sigma_{0 A B C} \frac{\left(\varepsilon_{A B C}^{F}\right)^{\alpha_{A B C}}}{1+\varepsilon_{A B C}^{F}} .
$$

$C_{A B C}$ is determined from the experimental condition of stress $\sigma_{0,2 A B C}$ in alloy in the form [9]

$$
C_{A B C}=\frac{f_{A B C}\left(\varepsilon_{0.2}\right)}{\sigma_{0.2 A B C} \varepsilon_{0.2}} .
$$

From the obtained value of $\varepsilon_{A B C}^{F}$, we can calculate $\sigma_{0 A B C}$ and $\alpha_{A B C}$. The limit of elastic deformation $\sigma_{e}=E_{A B C} \varepsilon_{e}$ of alloy $A B C$ is determined by [9]

$$
\sigma_{e}=E_{A B C} \varepsilon_{e}=\sigma_{0 A B C} \frac{\varepsilon_{e}^{\alpha}}{1+\varepsilon_{e}} .
$$

Here $E_{A B C}$ is Young's modulus of alloy $A B C$ and has the form $[5,6]$

$$
\begin{aligned}
E_{A B C}= & c_{B}\left(E_{B}-E_{A}\right)+E_{A C}, \\
E_{Y}= & \frac{1}{\pi \cdot r_{1 Y} \cdot A_{1 Y}}, \\
A_{1 Y}= & \frac{1}{k_{Y}}\left[1+\frac{2 \gamma_{Y}^{2} \theta^{2}}{k_{Y}^{4}}\left(1+\frac{1}{2} x_{Y} c t h x_{Y}\right)\left(1+x_{Y} c t h x_{Y}\right)\right], \quad x_{Y}=\frac{\hbar \omega_{Y}}{2 \theta}, Y=A, B, \\
E_{A C}= & E_{A}\left(1-15 c_{C}+c_{C} \frac{\left(\partial^{2} \psi_{C} / \partial \varepsilon^{2}\right)+6\left(\partial^{2} \psi_{A_{1}} / \partial \varepsilon^{2}\right)+8\left(\partial^{2} \psi_{A_{2}} / \partial \varepsilon^{2}\right)}{\left(\partial^{2} \psi_{A} / \partial \varepsilon^{2}\right)}\right), \\
\frac{\partial^{2} \psi_{X}}{\partial \varepsilon^{2}=} & \left\{\frac{1}{2} \frac{\partial^{2} U_{0 X}}{\partial r_{1 X}^{2}}+\frac{3}{4} \frac{\hbar \omega_{X}}{k_{X}}\left[\frac{\partial^{2} k_{X}}{\partial r_{1 X}^{2}}-\frac{1}{2 k_{X}}\left(\frac{\partial k_{X}}{\partial r_{1 X}}\right)\right]^{2}\right\} 4 r_{01 X}^{2}+ \\
& +\left(\frac{1}{2} \frac{\partial U_{0 X}}{\partial r_{1 X}}+x_{X} c t h x_{X} \frac{3 \theta}{2 k_{X}} \frac{\partial k_{X}}{\partial r_{1 X}}\right) 2 r_{01 X}, \quad x_{X}=\frac{\hbar \omega_{X}}{2 \theta}, \omega_{X}=\sqrt{\frac{k_{X}}{m_{X}}}, X=A, A_{1}, A_{2}, C .
\end{aligned}
$$


TABle 1: Mie-Lennard-Jones potential parameters for interactions $\mathrm{Au}-\mathrm{Au}, \mathrm{Cu}-\mathrm{Cu}$, and $\mathrm{Si}-\mathrm{Si}$.

\begin{tabular}{lcccc}
\hline Interaction & $D / k_{B}(\mathrm{~K})$ & $r_{0}\left(10^{-10} \mathrm{~m}\right)$ & $m$ & $n$ \\
\hline $\mathrm{Au}-\mathrm{Au} \mathrm{[27]}$ & 7411.5 & 2.8751 & 1.96 & 15.56 \\
$\mathrm{Cu}-\mathrm{Cu} \mathrm{[27]}$ & 6841.3 & 2.5487 & 3.03 & 8.37 \\
$\mathrm{Si}-\mathrm{Si}[25]$ & 32701.7 & 2.295 & 6 & 12 \\
\hline
\end{tabular}

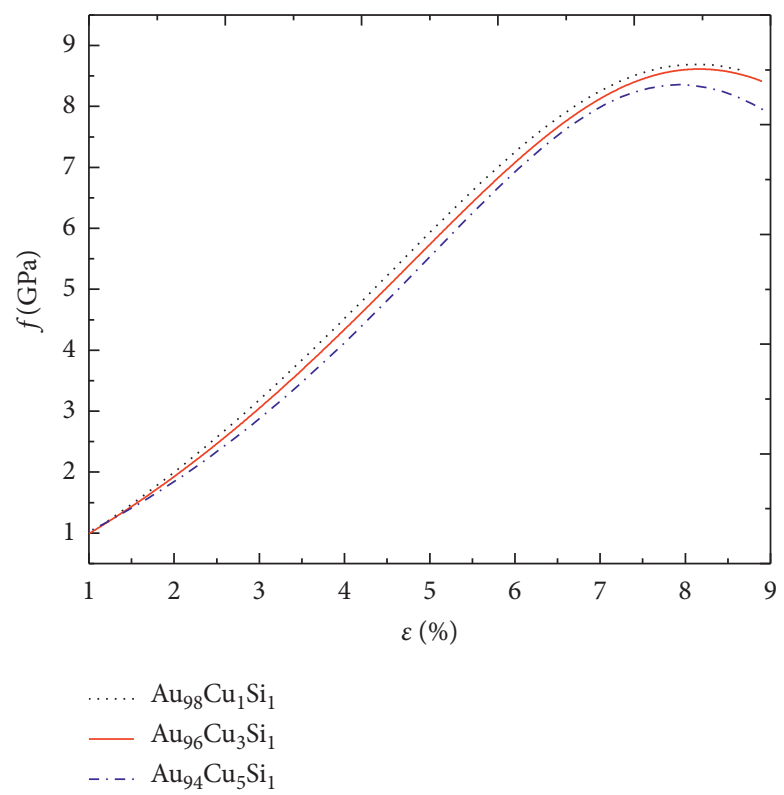

(a)

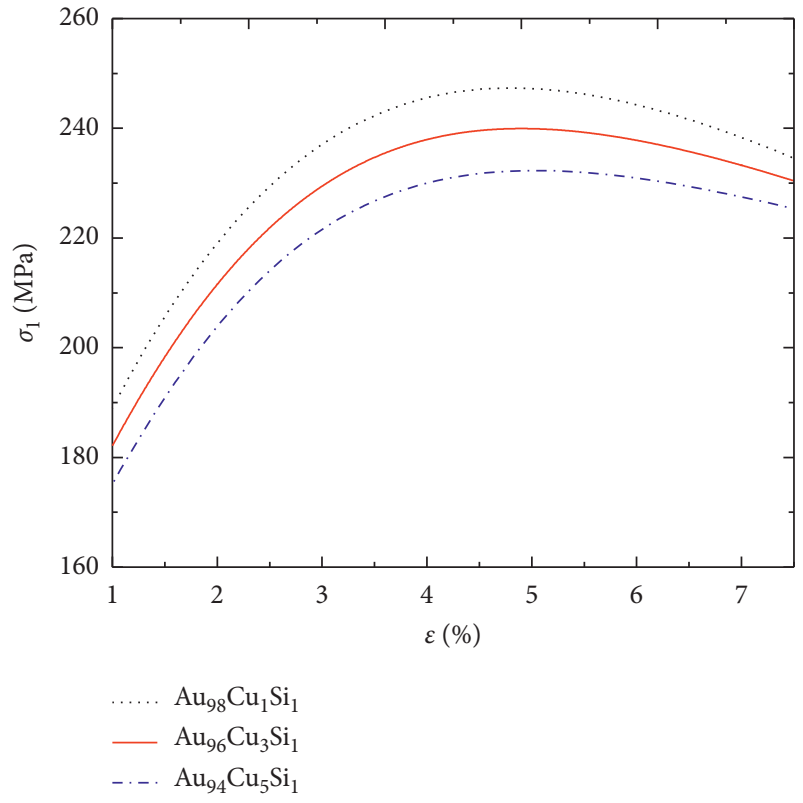

(b)

Figure 1: The density of deformation energy $f(\varepsilon)(\mathrm{a})$ and the real stress $\sigma_{1}(\varepsilon)(\mathrm{b})$ of $\mathrm{Au}_{99-x} \mathrm{Cu}_{x} \mathrm{Si}_{1}$ at $T=300 \mathrm{~K}$ and $P=0$ calculated by the SMM.

Here $E_{A C}, E_{A}, E_{B}$ are Young's moduli of alloy $A C$ and metals $A, B$, respectively.

\section{Numerical Results for Alloy AuCuSi}

To describe the interaction between atoms $\mathrm{Au}$ and $\mathrm{Si}$, we apply the Mie-Lennard-Jones pair interaction potential in the form [25]

$$
\varphi(r)=\frac{D}{n-m}\left[m\left(\frac{r_{0}}{r}\right)^{n}-n\left(\frac{r_{0}}{r}\right)^{m}\right],
$$

where $D$ is the depth of potential well corresponding to the equilibrium distance $r_{0}$ and $m$ and $n$ are determined empirically. Then, the potential parameters for the interaction $\mathrm{Au}-\mathrm{Si}$ are determined by [26]

$$
\begin{aligned}
& D_{\mathrm{Au}-\mathrm{Si}}=\sqrt{D_{\mathrm{Au}-\mathrm{Au}} D_{\mathrm{Si}-\mathrm{Si}}}, \\
& r_{0 \mathrm{Au}-\mathrm{Si}}=\frac{1}{2}\left(r_{0 \mathrm{Au}-\mathrm{Au}}+r_{0 \mathrm{Si}-\mathrm{Si}}\right) .
\end{aligned}
$$

We find $m_{\mathrm{Au}-\mathrm{Si}}$ and $n_{\mathrm{Au}-\mathrm{Si}}$ by fitting the theoretical result with the experimental data for Young's modulus of interstitial alloy $\mathrm{AuSi}_{3 \%}$ at room temperature. The Mie-LennardJones potential parameters for the interactions $\mathrm{Au}-\mathrm{Au}, \mathrm{Si}-\mathrm{Si}$, and $\mathrm{Au}-\mathrm{Si}$ are given in Table 1. The Poisson ratio is 0.42 for $\mathrm{Au}$ [28], 0.34 for $\mathrm{Cu}$ [29], and 0.21 for Si [30]. Our investigated range of temperature up to $900 \mathrm{~K}$ is below the melting temperature of $\mathrm{Au}, \mathrm{Cu}$, and $\mathrm{Si}$ in the range of pressure from zero to $6 \mathrm{GPa}$ as studies on the melting curve for these materials [31-33].

From

$$
E=\frac{\sigma_{0,2}}{\varepsilon_{0,2}} \Longrightarrow \sigma_{0,2}=E \varepsilon_{0,2}=E \times 0.2 \%,
$$

the experimental value of Young's modulus $E=89.1 .10^{9} \mathrm{~Pa}$ for $\mathrm{Au}$ at $T=300 \mathrm{~K}$ and $P=0$ [34]. From that, we obtain $\sigma_{0.2}=89.1 \times 10^{9} \times 0.002=178.2 \times 10^{6} \mathrm{~Pa}$. Figure 1 shows the density of deformation energy $f(\varepsilon)$ and the real stress $\sigma_{1}(\varepsilon)$ of AuCuSi at $T=300 \mathrm{~K}, P=0, c_{\mathrm{Si}}=1 \%$, and $c_{\mathrm{Cu}}=1,3$, and $5 \%$ calculated by the SMM. For AuCuSi at the same temperature, pressure, and concentration of interstitial atoms, when the concentration of substitutional atoms increases, the maximum real stress decreases by $2 \%$ and the elastic limit also decreases by $2 \%$. The density of deformation energy has the maximum value when the strain $\varepsilon_{F}=0.08$, and from that, we can find the maximum real stress $\sigma_{1 \text { max }}$, the elastic limit $\sigma_{e}$, and the elastic strain $\varepsilon_{e}$ for $\mathrm{AuCuSi}$ as shown in Table 2. 
TABle 2: Values of $\varepsilon_{F}\left(f\left(\varepsilon_{F}\right)=f_{\text {max }}\right), \sigma_{1 \max }, \sigma_{e}$, and $\varepsilon_{e}$ of $\mathrm{Au}_{99-x} \mathrm{Cu}_{x} \mathrm{Si}_{1}$ at $P=0$ and $T=300 \mathrm{~K}$ calculated by the SMM.

\begin{tabular}{lcccc}
\hline & $\varepsilon_{F}(\%)$ & $\sigma_{1 \max }(\mathrm{MPa})$ & $\varepsilon_{e}(\%)$ & $\sigma_{\mathrm{e}}(\mathrm{MPa})$ \\
\hline $\mathrm{Au}_{98} \mathrm{Cu}_{1} \mathrm{Si}_{1}$ & 8.0 & 231.79 & 0.21 & 190.84 \\
$\mathrm{Au}_{96} \mathrm{Cu}_{3} \mathrm{Si}_{1}$ & 8.0 & 230.38 & 0.21 & 189.40 \\
$\mathrm{Au}_{94} \mathrm{Cu}_{5} \mathrm{Si}_{1}$ & 8.0 & 227.06 & 0.20 & 186.38 \\
\hline
\end{tabular}

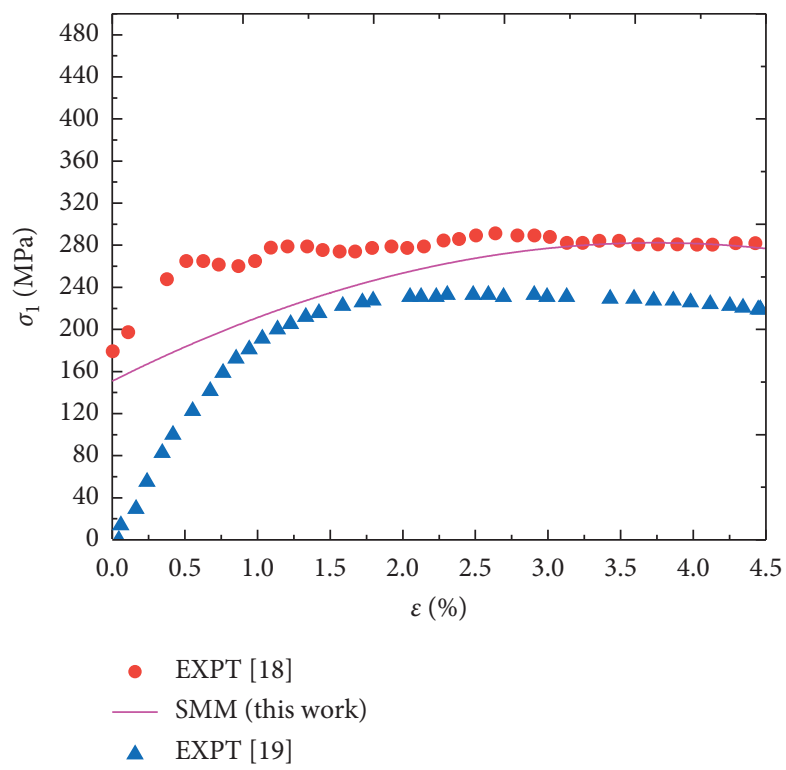

Figure 2: The real stress $\sigma_{1}(\varepsilon)$ of $\mathrm{Au}$ at $T=300 \mathrm{~K}$ and $P=0$ calculated by the SMM and from EXPT [11] and EXPT [12].

When concentrations $c_{\mathrm{Cu}}, c_{\mathrm{Si}} \longrightarrow 0$, we obtain the nonlinear deformation of main metal Au. Figure 2 shows the curve of real stress $\sigma_{1}(\varepsilon)$ for Au at $P=0$ and $T=300 \mathrm{~K}$ where we have the comparison between the SMM and experiments $[11,12]$. The maximum real stress $\sigma_{1 \max }$ and the elastic limit $\sigma_{e}$ calculated by the SMM in Table 3 are in good agreement with the molecular dynamics (MD) results [16] and experiments [13, 14]. The error of the maximum real stress between the SMM calculation and the $\mathrm{MD}$ result [16] is $0.6 \%$. The error of the elastic limit between the SMM calculation and the experimental data [13] is $5.65 \%$. Thus, the SMM calculations of the maximum real stress and the elastic limit for Au at $P=0$ and $T=300 \mathrm{~K}$ are in good agreement with other calculations and experiments.

Figure 3 shows the density of deformation energy $f(\varepsilon)$ and the real stress $\sigma_{1}(\varepsilon)$ of AuCuSi at $T=300 \mathrm{~K}, P=0$, $c_{\mathrm{Cu}}=10 \%$, and $c_{\mathrm{Si}}=0,1$, and $2 \%$ calculated by the SMM. For $\mathrm{AuCuSi}$ at the same temperature, pressure, and concentration of substitutional atoms, when the concentration of interstitial atoms increases, the maximum real stress and the elastic limit decrease strongly. We have the comparison on values of $\varepsilon_{F}, \sigma_{\text {lmax }}, \varepsilon_{e}$, and $\sigma_{e}$ for alloys $\mathrm{AuSi}, \mathrm{AuCu}$, and AuCuSi in Table 4.

Figure 4 shows the density of deformation energy $f(\varepsilon)$ and the real stress $\sigma_{1}(\varepsilon)$ of $\mathrm{AuCuSi}$ at $T=300,600$, and $900 \mathrm{~K}, P=0, c_{\mathrm{Cu}}=10 \%$, and $c_{\mathrm{Si}}=2 \%$ calculated by the SMM. For $\mathrm{AuCuSi}$ at the same pressure, concentration of
TABle 3: Values of $\varepsilon_{F}\left(f\left(\varepsilon_{F}\right)=f_{\max }\right), \sigma_{1 \max }, \varepsilon_{e}$, and $\sigma_{e}$ for Au at $P=0$ and $T=300 \mathrm{~K}$ calculated by the SMM and from MD [16], EXPT [13], and EXPT [14].

\begin{tabular}{|c|c|c|c|c|c|}
\hline \multicolumn{2}{|l|}{$\sigma_{\operatorname{lmax}}(\mathrm{MPa})$} & \multicolumn{4}{|c|}{$\sigma_{e}(\mathrm{MPa})$} \\
\hline SMM & $\begin{array}{l}\text { MD } \\
{[16]}\end{array}$ & SMN & & $\begin{array}{c}\text { EXPT } \\
{[13]}\end{array}$ & $\begin{array}{c}\text { EXPT } \\
{[14]}\end{array}$ \\
\hline$\varepsilon_{F}=9.6 \% \quad 243.52$ & 245 & $\varepsilon_{e}=0.19 \%$ & 188.70 & 200 & $45 \div 300$ \\
\hline
\end{tabular}

substitutional atoms, and concentration of interstitial atoms, when the temperature increases, the maximum real stress and the elastic limit increase. We have the comparison on values of $\varepsilon_{F}, \sigma_{1 \max }, \varepsilon_{e}$, and $\sigma_{e}$ for $\mathrm{Au}_{88} \mathrm{Cu}_{10} \mathrm{Si}_{2}$ at $P=0$ and $T=300,600$, and $900 \mathrm{~K}$ in Table 5 .

Figure 5 shows the real stress $\sigma_{1}(\varepsilon)$ of $\mathrm{AuCuSi}$ at $T=300 \mathrm{~K}, P=2,4$, and $6 \mathrm{GPa}, c_{\mathrm{Cu}}=10 \%$, and $c_{\mathrm{Si}}=3 \%$ calculated by the SMM. For AuCuSi at the same temperature, concentration of substitutional atoms, and concentration of interstitial atoms, when the pressure increases, the maximum real stress increases and the elastic limit decreases. When the pressure increases from 2 to $4 \mathrm{GPa}$, the maximum real stress increases by $3 \%$ and the elastic limit decreases by $7.86 \%$. When the pressure increases from 4 to $6 \mathrm{GPa}$, the maximum real stress increases by $11 \%$ and the elastic limit decreases by $10.56 \%$. We have the comparison on values of $\varepsilon_{F}, \sigma_{1 \max }, \varepsilon_{e}$, and $\sigma_{e}$ for $\mathrm{Au}_{87} \mathrm{Cu}_{10} \mathrm{Si}_{3}$ at $P=2,4$, and $6 \mathrm{GPa}$ and $T=300 \mathrm{~K}$ in Table 6 . 


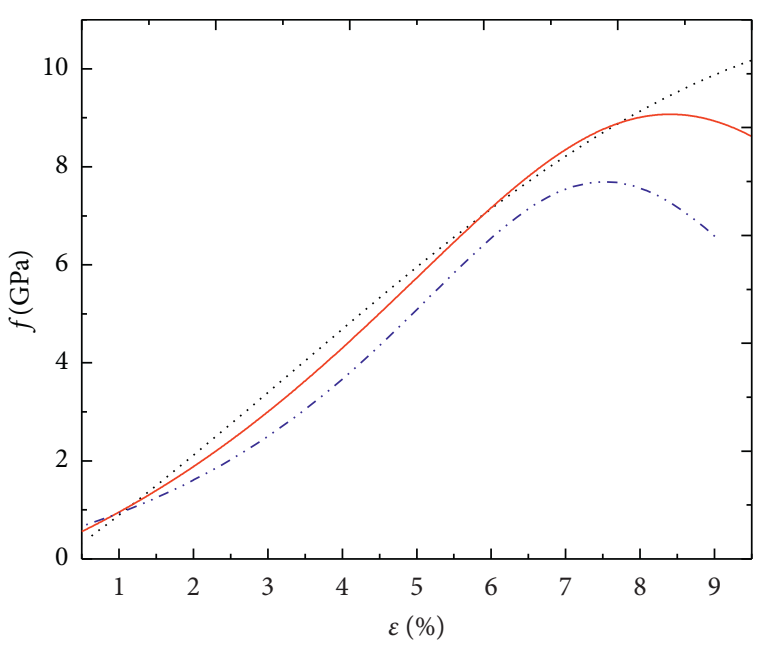

$$
\begin{aligned}
& \ldots . . \mathrm{Au}_{99} \mathrm{Si}_{1} \\
& \ldots \mathrm{Au}_{90} \mathrm{Cu}_{10} \\
& \ldots . . \mathrm{Au}_{89} \mathrm{Cu}_{10} \mathrm{Si}_{1}
\end{aligned}
$$

(a)

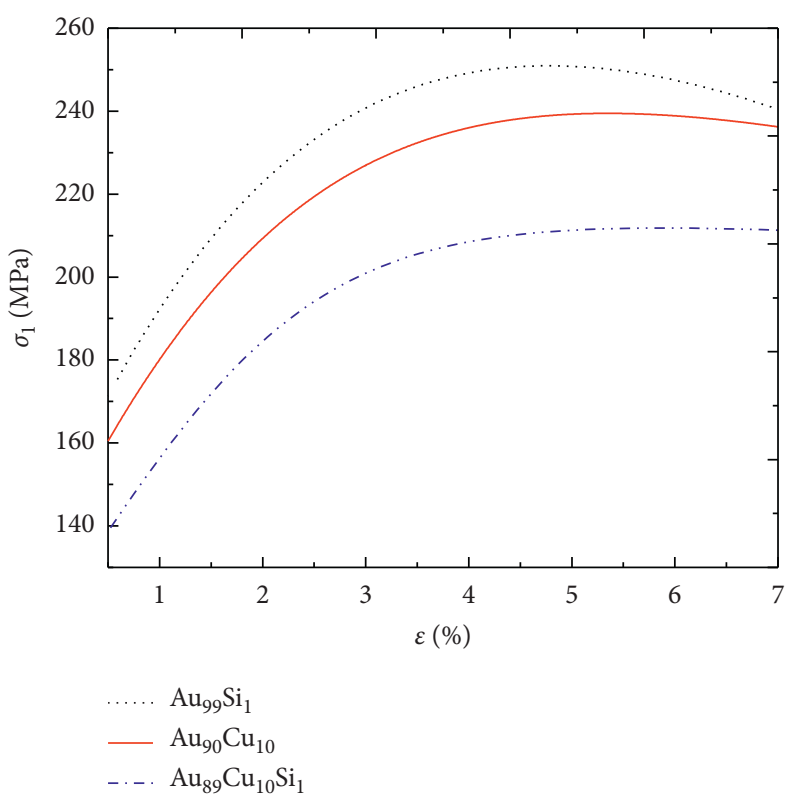

(b)

Figure 3: The density of deformation energy $f(\varepsilon)$ (a) and the real stress $\sigma_{1}(\varepsilon)(\mathrm{b})$ of $\mathrm{Au}_{99} \mathrm{Si}_{1}, \mathrm{Au}_{90} \mathrm{Cu}_{10}$, and $\mathrm{Au}_{89} \mathrm{Cu}_{10} \mathrm{Si}_{1}$ at $T=300 \mathrm{~K}$ and $P=0$ calculated by the SMM.

TABle 4: Values of $\varepsilon_{F}\left(f\left(\varepsilon_{F}\right)=f_{\max }\right), \sigma_{1 \max }, \varepsilon_{e}$, and $\sigma_{e}$ for $\mathrm{Au}_{99} \mathrm{Si}_{1}, \mathrm{Au}_{90} \mathrm{Cu}_{10}$, and $\mathrm{Au}_{89} \mathrm{Cu}_{10} \mathrm{Si}_{1}$ at $P=0$ and $T=300 \mathrm{~K}$ calculated by the $\mathrm{SMM}$.

\begin{tabular}{lcccc}
\hline Alloy & $\varepsilon_{F}(\%)$ & $\sigma_{1 \max }(\mathrm{MPa})$ & $\varepsilon_{e}(\%)$ & 0.18 \\
\hline $\mathrm{Au}_{99} \mathrm{Si}_{1}$ & 9.6 & 214.07 & 0.19 & 165.49 \\
$\mathrm{Au}_{90} \mathrm{Cu}_{10}$ & 8.3 & 242.95 & 0.19 & 196.75 \\
$\mathrm{Au}_{89} \mathrm{Cu}_{10} \mathrm{Si}_{1}$ & 8.2 & 216.79 & 175.98 \\
\hline
\end{tabular}

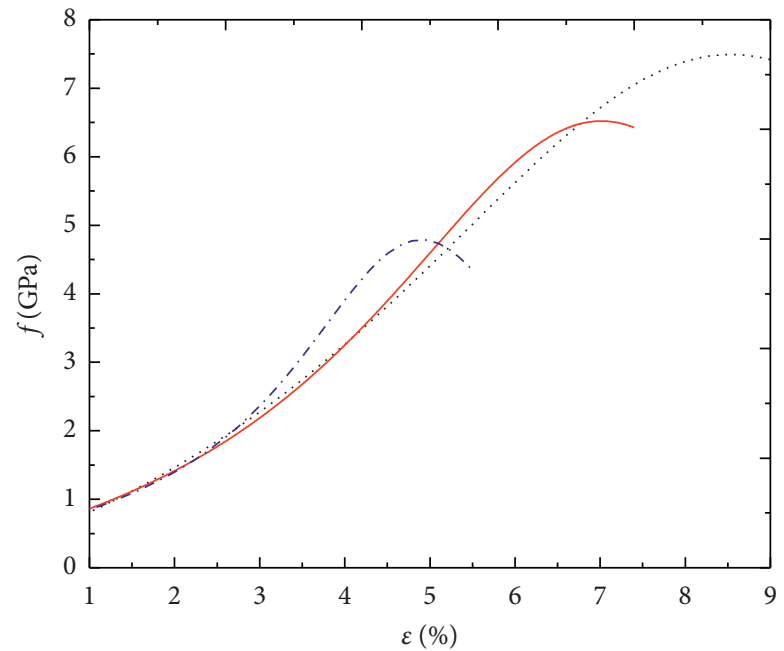

$\ldots 300 \mathrm{~K}$
$-\quad 600 \mathrm{~K}$
$\ldots .900 \mathrm{~K}$

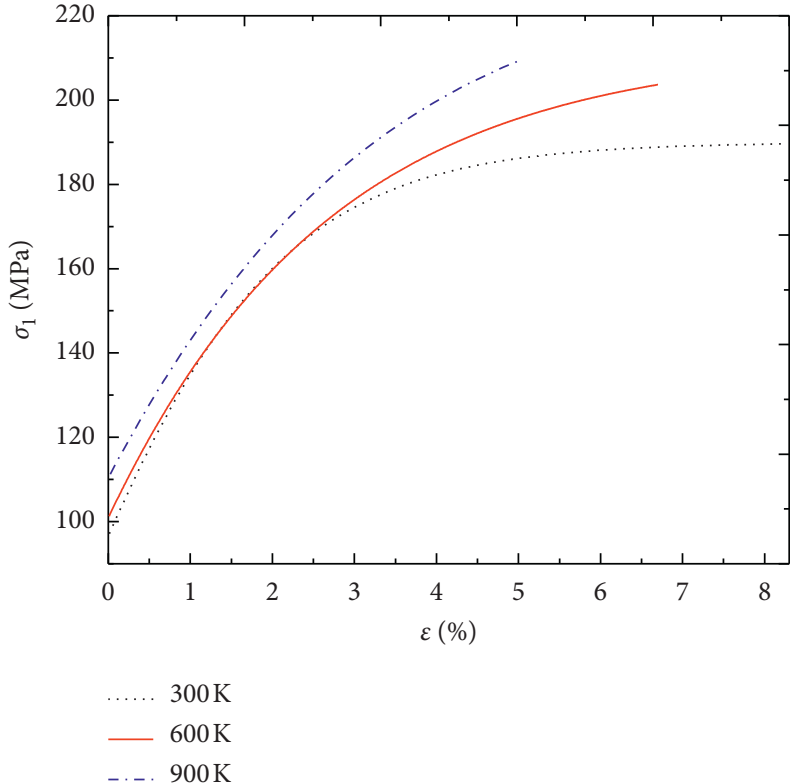

(b)

Figure 4: The density of deformation energy $f(\varepsilon)$ (a) and the real stress $\sigma_{1}(\varepsilon)$ (b) of $\mathrm{Au}_{88} \mathrm{Cu}_{10} \mathrm{Si}_{2}$ at $P=0$ and $T=300,600$, and $900 \mathrm{~K}$ calculated by the SMM. 
TABLe 5: Values of $\varepsilon_{F}\left(f\left(\varepsilon_{F}\right)=f_{\max }\right), \sigma_{1 \max }, \varepsilon_{e}$, and $\sigma_{e}$ for $\mathrm{Au}_{88} \mathrm{Cu}_{10} \mathrm{Si}_{2}$ at $P=0$ and $T=300,600$, and $900 \mathrm{~K}$ calculated by the SMM.

\begin{tabular}{lcccc}
\hline$T(\mathrm{~K})$ & $\varepsilon_{F}(\%)$ & $\sigma_{1 \max }(\mathrm{MPa})$ & $\varepsilon_{e}(\%)$ & \\
\hline 300 & 8.2 & 194.52 & 0.18 & \\
600 & 6.7 & 208.29 & 0.24 & 157.36 \\
900 & 5.0 & 210.27 & 0.32 & 179.85 \\
\hline
\end{tabular}

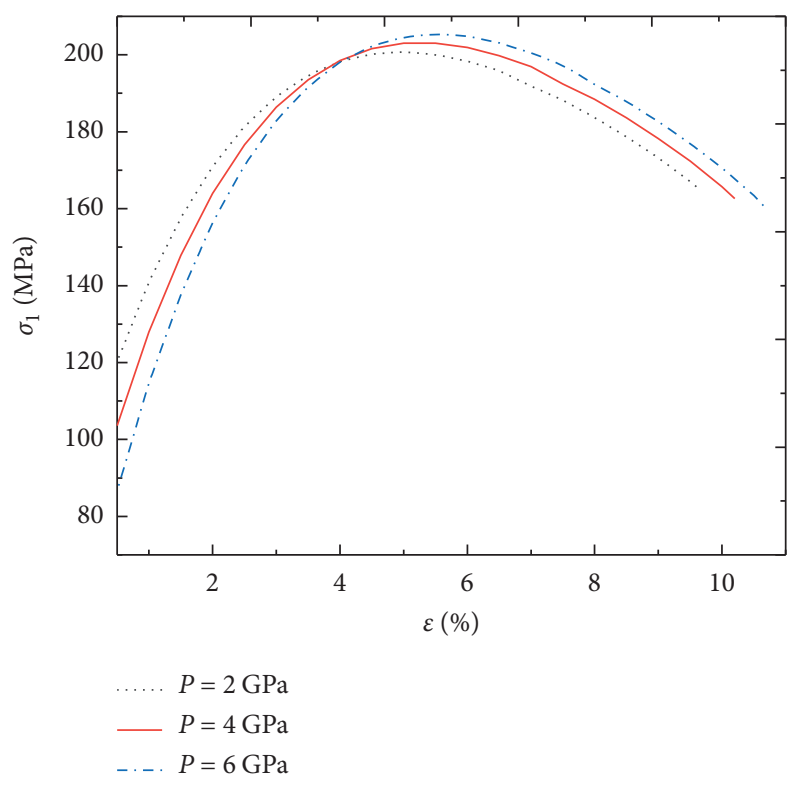

Figure 5: The real stress $\sigma_{1}(\varepsilon)$ of $\mathrm{Au}_{87} \mathrm{Cu}_{10} \mathrm{Si}_{3}$ at $P=2,4$, and $6 \mathrm{GPa}$ and $T=300 \mathrm{~K}$ calculated by the SMM.

TABLE 6: Values of $\varepsilon_{F}\left(f\left(\varepsilon_{F}\right)=f_{\max }\right), \sigma_{1 \max }, \varepsilon_{e}$, and $\sigma_{e}$ for $\mathrm{Au}_{88} \mathrm{Cu}_{10} \mathrm{Si}_{2}$ at $P=2,4$, and $6 \mathrm{GPa}$ and $T=300 \mathrm{~K}$ calculated by the SMM.

\begin{tabular}{lcccc}
\hline$P(\mathrm{GPa})$ & $\varepsilon_{F}(\%)$ & $\sigma_{1 \max }(\mathrm{MPa})$ & $\varepsilon_{e}(\%)$ & $\sigma_{e}(\mathrm{MPa})$ \\
\hline 2 & 8.9 & 175.86 & 0.14 & 149.50 \\
4 & 9.7 & 170.64 & 0.10 & 137.75 \\
6 & 10.2 & 152.06 & 0.08 & 123.20 \\
\hline
\end{tabular}

\section{Conclusion}

In our paper, we derive the analytic expressions of characteristic quantities for the nonlinear deformation such as the density of deformation energy, the maximum real stress, and the limit of elastic deformation depending on temperature, pressure, and concentration of components together with the strain-stress of substitutional alloy $A B$ with interstitial atom $C$ and FCC structure under pressure. We apply theoretical results to alloy $\mathrm{AuCuSi}$. The maximum real stress $\sigma_{1 \max }$ and the elastic limit $\sigma_{e}$ calculated by the SMM are in good agreement with MD results [16] and experiments $[13,14]$. For AuCuSi at the same temperature, concentration of substitutional atoms, and concentration of interstitial atoms, when the pressure increases, the maximum real stress increases and the elastic limit decreases. When the pressure increases from 2 to $4 \mathrm{GPa}$, the maximum real stress increases by $3 \%$ and the elastic limit decreases by $7.86 \%$. When the pressure increases from 4 to
$6 \mathrm{GPa}$, the maximum real stress increases by $11 \%$ and the elastic limit decreases by $10.56 \%$. Our calculated results for alloy $\mathrm{AuCuSi}$ are compared with ones for alloys $\mathrm{AuCu}, \mathrm{AuSi}$, and metal Au. If we use more coordination spheres and we have exact experimental data for the stress $\sigma_{0.2}$ of alloy $\mathrm{AuCuSi}$ at different temperatures, pressures, and concentrations of components, we will obtain better calculations.

\section{Data Availability}

The data that support the findings of this study are available from the corresponding author upon reasonable request.

\section{Conflicts of Interest}

The authors declare that they have no conflicts of interest.

\section{References}

[1] A. Smirnov, Theory Of Interstitial Alloys, Nauka, Moscow, Russia, in Russian, 1979.

[2] P. A. Korzhavyi, I. A. Abrikosov, B. Johansson, A. V. Ruban, and H. L. Skriver, "First-principles calculations of the vacancy formation energy in transition and noble metals," Physical Review B, vol. 59, no. 18, p. 11693, 1999.

[3] T. T. Lau, C. J. Först, X. Lin, J. G. Gale, S. Yip, and K. J. Van Vliet, Physical Review Letter, vol. 98, Article ID 215501, 2007. 
[4] M. Li, "Defect-induced topological order-to-disorder transition in two-dimensional binary substitutional solid solutions," Physical Review B, vol. 62, Article ID 13979, 2000.

[5] N. Q. Hoc and N. D. Hien, " $42^{\text {nd }}$ vietnam national Conference on theoretical physics (NCTP-42)," IOP Conference Series: Journal of Physics: Conference Series, vol. 1034, Article ID 012005, 2018.

[6] N. Q. Hoc, B. D. Tinh, and N. D. Hien, "Elastic moduli and elastic constants of alloy aucusi with fcc structure under pressure," High Temperature Materials and Processes, vol. 38, pp. 264-272, 2018.

[7] B. D. Tinh, N. Q. Hoc, D. Q. Vinh, T. D. Cuong, and N. D. Hien, Advances in Materials Science and Engineering, vol. 2018, p. 8, Article ID 5251741, 2018.

[8] N. Q. Hoc, T. D. Cuong, and N. D. Hien, "Proceedings of the ACCMS-theme meeting on "Multiscale modelling of materials for sustainable development"' VNU Journal of Sciences: Mathematics-Physics, vol. 35, no. 1, pp. 1-12, 2019.

[9] N. Q. Hoc, B. D. Tinh, and N. D. Hien, Romanian Journal of Physics, vol. 65, no. 5-6, p. 608, 2020.

[10] N. Q. Hoc, N. T. Hoa, T. D. Cuong, and D. Q. Thang, "On the melting of interstitial alloys $\mathrm{FeH}, \mathrm{FeSi}$ and $\mathrm{FeC}$ with a bodycentered cubic structure under pressure Proceedings of the ACCMS-theme meeting on "multiscale modelling of materials for sustainable development"' Vietnam Journal of Science, Technology and Engineering, vol. 61, no. 2, pp. 17-22, 2019.

[11] R. D. Nyilas, M. Kobas, and R. Spolenak, "Synchrotron X-ray microdiffraction reveals rotational plastic deformation mechanisms in polycrystalline thin films," Acta Materialia, vol. 57, no. 13, pp. 3738-3753, 2009.

[12] I. Chasiotis, C. Bateson, K. Timpano, A. S. McCarty, N. S. Barker, and J. R. Stanec, "Strain rate effects on the mechanical behavior of nanocrystalline Au films," Thin Solid Films, vol. 515, no. 6, pp. 3183-3189, 2007.

[13] https://structx.com/Material_Properties_003a.html.

[14] https://www.azom.com/properties.aspx?ArticleID=600.

[15] M. J. Mehl and D. A. Papaconstantopoulos, "Applications of a tight-binding total-energy method for transition and noble metals: elastic constants, vacancies, and surfaces of monatomic metals," Physical Review B, vol. 54, no. 7, p. 4519, 1996.

[16] M. N. Esfahani and M. Jabbari, "Molecular dynamics simulations of deformation mechanisms in the mechanical response of nanoporous gold," Materials, vol. 13, no. 9, p. 2071, 2020.

[17] N. T. Dung, N. C. Cuong, and D. Q. Van, Journal of Multiscale Modelling, vol. 11, no. 2, Article ID 2030001, 2020.

[18] N. T. Dung and N. T. Phuong, "Molecular dynamics study on factors influencing the structure, phase transition and crystallization process of NiCu6912 nanoparticle," Materials Chemistry and Physics, vol. 250, Article ID 123075, 2020.

[19] T. Q. Tuan and N. T. Dung, "Effect of heating rate, impurity concentration of $\mathrm{Cu}$, atomic number, temperatures, time annealing temperature on the structure, crystallization temperature and crystallization process of Ni1-xCux bulk; $x=0.1$, 0.3, 0.5, 0.7," International Journal of Modern Physics B, vol. 32, no. 26, p. 1830009, 2018.

[20] C. L. Van, D. Q. Van, and N. T. Dung, "Ab initio calculations on the structural and electronic properties of AgAu alloy," ACS Omega, vol. 5, no. 48, pp. 31391-31397, 2020.

[21] N. Tang and V. V. Hung, "Investigation of the thermodynamic properties of anharmonic crystals by the momentum method, I. General results for face-centered cubic crystals," Physica Status Solidi (B), vol. 149, p. 511, 1989.
[22] N. Tang and V. Van Hung, "Investigation of the thermodynamic properties of anharmonic crystals by the momentum method. II. Comparison of calculations with experiments for inert gas crystals," Physica Status Solidi (B), vol. 161, no. 1, p. $165,1990$.

[23] N. Tang and V. Van Hung, "Investigation of the thermodynamic properties of anharmonic crystals by the momentum method. III. Thermodynamic properties of the crystals at various pressures," Physica Status Solidi (B), vol. 162, no. 2, p. $371,1990$.

[24] N. Tang and V. Van Hung, "Investigation of the thermodynamic properties of anharmonic crystals by momentum method. IV. The limiting of absolute stability and the melting temperature of crystals," Physica Status Solidi (B), vol. 162, no. 2, p. 379, 1990.

[25] M. N. Magomedov, "On calculating the Debye temperature and the Gruneisen parameter," Zhurnal Fizicheskoi Khimii, vol. 61, no. 4, pp. 1003-1009, 1987, in Russian.

[26] R. J. Good and C. J. Hope, "New combining rule for intermolecular distances in intermolecular potential functions," The Journal of Chemical Physics, vol. 53, no. 2, pp. 540-543, 1970.

[27] M. N. Magomedov, "The calculation of the parameters of the Mie-Lennard-Jones potential," High Temperature, vol. 44, no. 4, pp. 513-529, 2006.

[28] E. Goens, Physikal. Z.vol. 37, p. 321, 1936.

[29] F. Birch, "Finite elastic strain of cubic crystals," Physical Review, vol. 71, no. 11, p. 809, 1947.

[30] B. Bayram, O. Akar, and T. Akin, "Plasma-activated direct bonding of diamond-on-insulator wafers to thermal oxide grown silicon wafers," Diamond and Related Materials, vol. 19, no. 11, pp. 1431-1435, 2010.

[31] Z. Y. Zhong, H. Saka, T. H. Kim, E. A. Holm, Y. K. Han, and X. S. Xie, "Effect of pressure on melting temperature of silicon and germanium," Materials Science Forum, vol. 475-479, pp. 1893-1896, 2005.

[32] D. Errandonea, Journal of Applied Physics, vol. 108, Article ID 033517, 2010.

[33] D. Errandonea, "High-pressure melting curves of the transition metals $\mathrm{Cu}, \mathrm{Ni}, \mathrm{Pd}$ and Pt," Physical Review B, vol. 87, Article ID 054108, 2013.

[34] L. V. Tikhonov and G. I. Kononenko, Mechanical Properties of Metals and Alloys, Kiev-Nauka Dumka, Ukraine, 1986. 